05,09

\title{
Инфракрасные спектры диффузного отражения микропорошков $\mathrm{Ni}_{1-x} \mathrm{Zn}_{x} \mathrm{Fe}_{2} \mathrm{O}_{4}$-ферритов
}

\author{
() Ш.Н. Алиева, А.М. Керимова , Р.Б. Абдуллаев, Т.Р. Мехтиев \\ Институт физики им. Г.М. Абдуллаева НАН Азербайджана, \\ Баку, Азербайджан \\ "E-mail: afet.kerimova@physics.ab.az
}

(Поступила в Редакцию 29 декабря 2015 г.

В окончательной редакции 7 августа 2016 г.)

\begin{abstract}
Представлены результаты экспериментальных исследований спектров диффузного отражения в инфракрасном диапазоне от 4000 до $50 \mathrm{~cm}^{-1}$ ) микропорошков $\mathrm{Ni}_{1-x} \mathrm{Zn}_{x} \mathrm{Fe}_{2} \mathrm{O}_{4}$-ферритов с различным содержанием $\mathrm{Ni}$ и $\mathrm{Zn}$. Установлена зависимость интенсивности ИК-спектров этих ферритов от изменения концентраций катионов $\mathrm{Fe}^{2+}$ и $\mathrm{Fe}^{3+}$ в разных составах ферритов. Исследованы особенности изменения спектров отражения в зависимости от составов ферритов, и дана предположительная интерпретация наблюдаемых спектральных структур.
\end{abstract}

Работа выполнена при финансовой поддержке Фонда развития науки при Президенте Азербайджанской республики. Грант № EİF02013-9(15)-46/05/1.

DOI: 10.21883/FTT.2017.03.44164.468

\section{1. Введение}

Несмотря на свою широкую известность, возникшую еще в прошлом столетии вследствие большой практической значимости, $\mathrm{Ni}_{1-x} \mathrm{Zn}_{x} \mathrm{Fe}_{2} \mathrm{O}_{4}$-ферриты по-прежнему вызывают большой научный интерес, что заметно по неуменьшающемуся объему научных публикаций. Характерными особенностями этих ферритов [1] является наличие двух магнитных подрешеток, связанных косвенным обменным взаимодействием (дипольные взаимодействия между атомами каждой из подрешеток много меньше, чем обменное взаимодействие между атомами разных подрешеток). Другой особенностью изученных составов ферритов является постепенное (по мере изменения содержания $\mathrm{Zn}$ ) преобразование структуры обращенной шпинели $\left(\mathrm{NiFe}_{2} \mathrm{O}_{4}\right)$ в структуру нормальной шпинели $\left(\mathrm{ZnFe}_{2} \mathrm{O}_{4}\right)$ без изменения симметрии, но наблюдается переход от ферримагнитного упорядочения в антиферромагнитное. Заметим, что начальная магнитная проницаемость ферритов по мере увеличения содержания в них цинка (для $\mathrm{NiFe}_{2} \mathrm{O}_{4} \mu_{\mathrm{H}}=55$ ) возрастает, достигая максимума $\left(\mu_{\mathrm{H}}=4200\right)$ в составе $\mathrm{Ni}_{0.28} \mathrm{Zn}_{0.72} \mathrm{Fe}_{2} \mathrm{O}_{4}$ и далее резко уменьшается [2]. В работах $[3,4]$, посвященных исследованиям малоуглового рассеяния нейтронов от поликристаллических образцов $\mathrm{Ni}_{1-x} \mathrm{Zn}_{x} \mathrm{Fe}_{2} \mathrm{O}_{4}$-ферритов, синтезированных по обычной керамической технологии, было показано, что в составах ферритов с $x=0.60,0.68,0.75$ при низких температурах регистрируется высокополевая восприимчивость, характерная для неколлинеарной магнитной структуры. В этих составах при температуре $4.2 \mathrm{~K}$ возникало интенсивное малоугловое рассеяние, указывающее на появление магнитных неоднородностей (средние размеры: от 1 до $10 \mathrm{~nm})$ в $z$-проекции магнитных моментов. В работе [4] было показано, что в феррите
$\mathrm{Ni}_{0.4} \mathrm{Zn}_{0.6} \mathrm{Fe}_{2} \mathrm{O}_{4}$ неоднородная магнитная структура существует во всем температурном интервале ниже температуры Кюри и является следствием конкурирующих межподрешеточных и внутриподрешеточных обменных взаимодействий. В то же время, неоднородная магнитная структура является причиной возникновения в этом феррите повышенной плотности состояний длинноволновых магнитных возбуждений. Как показали исследования мессбауэровских спектров, в микропорошках магнетита (размер зерен от 15 до $45 \mathrm{~nm}$ ), структурного аналога $\mathrm{Ni}_{1-x} \mathrm{Zn}_{x} \mathrm{Fe}_{2} \mathrm{O}_{4}$-ферритов, сушествует слабомагнитная фаза [5], параметры которой определить не удалось ввиду малой интенсивности соответствующих пиков и невозможности разделить их от интенсивных максимумов ионов железа $A$ - и $B$-подрешеток. Как считают авторы статьи, можно лишь упоминать о суперпарамагнетизме этой фазы. В исследованиях на атомном силовом микроскопе магнитных профилей тонких (порядка $\sim 100 \mathrm{~nm}$ ) пленок с разным содержанием $\mathrm{Ni}$ и Zn при температуре $300 \mathrm{~K}$ была обнаружена магнитная доменная структура этих ферритов [6], причем размеры доменов для разных составов $\mathrm{Ni}_{1-x} \mathrm{Zn}_{x} \mathrm{Fe}_{2} \mathrm{O}_{4}$ менялись соответственно изменениям содержания в них катионов $\mathrm{Fe}$.

Как известно [7], магнитные спектры доступны наблюдению в опытах по неупругому рассеянию нейтронов, в опытах по инфракрасному и комбинационному рассеяниям и магнитному резонансу. Описание неупругого рассеяния и комбинационного рассеяния формально аналогично описанию аналогичных процессов с участием фононов.

В данной публикации представлены результаты экспериментальных исследований влияния магнитных неоднородностей на ИК-спектры микропорошков $\mathrm{Ni}_{1-x} \mathrm{Zn}_{x} \mathrm{Fe}_{2} \mathrm{O}_{4}$-ферритов с разным содержанием $\mathrm{Ni}$ и $\mathrm{Zn}$. 
Частоты фононов $\mathrm{Ni}_{1-x} \mathrm{Zn}_{x} \mathrm{Fe}_{2} \mathrm{O}_{4}$ в области от $50 \mathrm{~cm}^{-1}$ до $700 \mathrm{~cm}^{-1}$ ИК-спектра

\begin{tabular}{|c|c|c|c|c|c|c|c|c|c|c|c|c|}
\hline \multirow{4}{*}{$\begin{array}{c}\text { Тип } \\
\text { симметрии }\end{array}$} & \multicolumn{12}{|c|}{ Литературные ссылки и полученные экспериментальные результаты } \\
\hline & {$[12],[13]$} & \multicolumn{2}{|c|}{ эксперимент } & [12] & \multicolumn{2}{|c|}{ эксперимент } & [12] & эксперимент & [11], [12] & \multicolumn{2}{|c|}{ эксперимент } & {$[9],[12]$} \\
\hline & \multicolumn{12}{|c|}{$x$} \\
\hline & 0 & 0 & 0.25 & 0.3 & 0.4 & 0.5 & 0.5 & 0.6 & 0.7 & 0.75 & 1.0 & 1.0 \\
\hline \multirow[t]{8}{*}{$F_{1 u}^{1}$} & $\begin{array}{l}604 \\
{[12]}\end{array}$ & 604 & 592 & $\begin{array}{l}590 \\
{[12]}\end{array}$ & 584 & 578 & $\begin{array}{l}578 \\
{[12]}\end{array}$ & 582 & $\begin{array}{l}571 \\
{[12]} \\
582 \\
{[11]}\end{array}$ & 570 & - & $\begin{array}{l}569 \\
{[9]}\end{array}$ \\
\hline & & - & - & & - & 544 & & 544 & & 550 & $\begin{array}{l}542 \\
{[12]} \\
\end{array}$ & 542 \\
\hline & & 533 & 538 & & 522 & & & 535 & & - & - & \\
\hline & & 529 & 528 & & 512 & 529 & & 525 & & 516 & 519 & \\
\hline & & - & 524 & & 508 & - & & 518 & & 506 & 507 & \\
\hline & $\begin{array}{l}460 \\
{[12]}\end{array}$ & - & 456 & & 497 & 454 & & - & & 500 & 471 & $\begin{array}{l}463 \\
{[12]}\end{array}$ \\
\hline & & 443 & 442 & & 437 & 436 & & - & & - & - & \\
\hline & & 432 & 433 & & & 429 & & 430 & & - & - & \\
\hline \multirow[t]{7}{*}{$F_{1 u}^{2}$} & $\begin{array}{l}426 \\
{[12]}\end{array}$ & 425 & 426 & $\begin{array}{l}426 \\
{[12]}\end{array}$ & 426 & 424 & $\begin{array}{l}426 \\
{[12]}\end{array}$ & - & $\begin{array}{l}426 \\
{[12]}\end{array}$ & 421 & 426 & $\begin{array}{c}420 \\
{[9]}\end{array}$ \\
\hline & & - & 403 & & 401 & - & & 403 & $\begin{array}{l}401 \\
{[11]}\end{array}$ & 394 & 398 & \\
\hline & & 392 & 391 & & 389 & 389 & & 388 & & 391 & 388 & \\
\hline & $\begin{array}{l}373 \\
{[13]}\end{array}$ & - & 367 & & 363 & 356 & & - & & 330 & 332 & \\
\hline & & 349 & 345 & & 346 & 343 & & 324 & & - & - & \\
\hline & & 306 & 306 & & 304 & 300 & & 299 & & 308 & 313 & \\
\hline & $\begin{array}{l}273 \\
{[13]}\end{array}$ & 273 & 275 & & 270 & 266 & & 284 & & 287 & 294 & \\
\hline \multirow[t]{7}{*}{$F_{1 u}^{3}$} & $\begin{array}{l}250 \\
{[13]}\end{array}$ & 249 & 247 & & - & 248 & & 236 & & - & 247 & \\
\hline & & - & 204 & & 204 & 206 & & 195 & & 206 & 206 & $\begin{array}{l}206 \\
{[12]}\end{array}$ \\
\hline & & 169 & 169 & & 171 & 173 & & 163 & & 177 & 183 & \\
\hline & & 96 & 93 & & 95 & 95 & & 128 & & 81 & 84 & \\
\hline & & 95 & 90 & & 89 & 88 & & 55 & & 77 & 80 & \\
\hline & & - & 85 & & 84 & 83 & & 52 & & 73 & 76 & \\
\hline & & 74 & 76 & & 71 & 72 & & 43 & & 58 & 69 & \\
\hline
\end{tabular}

\section{2. Приготовление образцов}

Все составы ферритов были получены путем высокотемпературного синтеза сверхчистых порошков соединений $\mathrm{ZnO}, \mathrm{NiO}, \mathrm{Fe}_{2} \mathrm{O}_{3}$ [2,7]. Детально, технология синтеза ферритов $\mathrm{Ni}_{1-x} \mathrm{Zn}_{x} \mathrm{Fe}_{2} \mathrm{O}_{4}$ опубликована в работе [2].
Как известно [2], $\mathrm{Ni}_{1-x} \mathrm{Zn}_{x} \mathrm{Fe}_{2} \mathrm{O}_{4}$-ферриты не растворяют избыточного количества $\mathrm{NiO}$ и $\mathrm{ZnO}$. Избыточное количество $\mathrm{Fe}_{2} \mathrm{O}_{3}$ приводит к образованию твердого раствора, содержащего магнетит $\mathrm{Fe}_{3} \mathrm{O}_{4}$. Как указано в [2], для достижения устойчивого состояния в шпинельных структурах всегда возникают различные формы беспо- 
рядка в виде точечных дефектов и вакансий, устойчивость и концентрация которых практически не меняется до тех пор, пока термодинамическое равновесие не будет нарушено и для обеспечения полноты протекания реакции образования ферритов в стадии спекания смесь окислов необходимо отжигать при температурах от 900 до $1000^{\circ} \mathrm{C}$.

В настоящей работе все синтезированные составы $\mathrm{Ni}_{1-x} \mathrm{Zn}_{x} \mathrm{Fe}_{2} \mathrm{O}_{4}$-ферритов (=0, 0.25, 0.4, 0.5, 0.6, 0.75, 1) обжигались при температуре $960^{\circ} \mathrm{C}$. Далее они измельчались до микропорошков с размерами зерен порядка $20 \mathrm{~nm}$.

Качество синтезированных составов микропорошков ферритов контролировалось по рентгеновским дифрактограммам на XRDD8 ADVANCE (Bruker, Germany), a также по Раман-спектрам. Результаты рентгеновских исследований всех синтезированных составов ферритов имели структуру шпинели с пространственной группой симметрии $F d \overline{3} m[1]$ и были ранее опубликованы в работе [8]. Были определены параметры элементарных ячеек и показано, что все составы $\mathrm{Ni}_{1-x} \mathrm{Zn}_{x} \mathrm{Fe}_{2} \mathrm{O}_{4}$ сохраняют свою структуру вплоть до $1100^{\circ} \mathrm{C}$.

Инфракрасные спектры диффузного отражения $\mathrm{Ni}_{1-x} \mathrm{Zn}_{x} \mathrm{Fe}_{2} \mathrm{O}_{4}$ исследовались на инфракрасном Фурьеспектрометре Vertex70 (Bruker, Germany) в вакуумной камере со стандартной приставкой диффузного отражения EasiDiff ${ }^{\mathrm{TM}}$ (PIKETechnologies, USA) в спектральном диапазоне от 4000 до $50 \mathrm{~cm}^{-1}$, стандартное спектральное разрешение - лучше $0.5 \mathrm{~cm}^{-1}$ и показаны на рис. 1,2 . Температура образцов во всех проведенных исследованиях была равной $300 \mathrm{~K}$. Положения максимумов спектров диффузного отражения, в программном обеспечении „OPUS“ Фурье-спектрометра анализируются уравнением Кубелки-Мунка, которое для толстого, непрозрачного слоя мелкого порошка толщиной в несколько миллиметров, имеет вид $F(R) \equiv \frac{(1-R)}{2 R}=\frac{k}{s}$, где $R=\Phi_{1} / \Phi_{2} ; \Phi_{1}-$ диффузное отражение слоя порошка, $\Phi_{2}$ - диффузное отражение непоглощающего эталона; $k$ - молярный коэффициент поглощения, определяется законом Ламберта: $\Phi=\Phi_{0} \exp (-k d) ; s-$ коэффициент рассеяния, который предполагается не зависящим от длины волны. В качестве эталона использовался стандартный эталон (PIKE Technologies) чистый карбид кремния. Положения максимумов поглощения (определяются из уравнения Кубелки-Мунка) исследуемых составов $\mathrm{Ni}_{1-x} \mathrm{Zn}_{x} \mathrm{Fe}_{2} \mathrm{O}_{4}$, а также наличие тонкой структуры, приведены в таблице и были установлены в результате многократно проведенных экспериментов и согласуются с результатами экспериментальных работ [9-13], которые вместе с полученными в настоящей работе данными приведены в таблице. Более детальная обработка ИК-спектров проводилась методом Крамерса-Кронига (эта процедура включена в программное обеспечение Фурье-спектрометра и устанавливает связь между спектрами отражения и поглощения, а также спектрами преломления, мнимой и реальной частями диэлектрической проницаемости, функцией потерь).
При анализе полученных ИК-спектров было установлено, что профили их имеют сложную структуру и в процессе изменения $x$, заметен не только сдвиг, но и расщепления на спектральные компоненты.

\section{3. Экспериментальные результаты и обсуждение}

Хорошо известно, что в инфракрасных спектрах отражения шпинелей пространственной группы симметрии - $F d \overline{3} m[3]$, должны наблюдаться ИК-активные трехкратно вырожденные моды $F_{1 u}$-типа симметрии, то есть колебания асимметричные относительно центра инверсии и симметричные относительно оси второго порядка или вертикальных плоскостей отражения [14]. Поскольку массы ионов $\mathrm{Fe}$ (55.847), $\mathrm{Ni}$ (58.69) и $\mathrm{Zn}$ (65.39), входящих в состав исследуемого феррита, намного превышают массу иона кислорода (15.999), колебания ионов кислорода относительно тяжелых ионов металлов практически не влияют на положения тяжелых ионов, в то время как колебания тяжелых ионов, естественно, влияют на колебания иона кислорода. Смещение иона кислорода может происходить либо вдоль оси третьего порядка $C_{3}$, либо перпендикулярно к ней [12]. В первом случае наблюдается колебание $F_{1 u}^{1}$ связи $M e^{2+}-\mathrm{O}-3 M e^{3+}$ (где $M e^{2+}-$ октаэдрический катион, $M e^{3+}$ - три тетраэдрических катиона). При смещении кислорода перпендикулярно оси $C_{3}$ наблюдается колебание $F_{1 u}^{2}$ связи $M e^{2+}-\mathrm{O}-3 M e^{3+}$. В общем случае, этим колебаниям соответствуют соответственно высокочастотная и низкочастотная полосы спектра. Колебания катионов относительно друг друга $F_{1 u}^{3}$ (связь $\left.M e^{3+}-M e^{3+}\right)$ типа симметрии происходят при еще более низких частотах и имеют слабые интенсивности [12].

Как показано в работе [15], слабо интенсивная, широкая полоса поглощения в области $3627-3500 \mathrm{~cm}^{-1}$ (рис. 2) соответствует вкладу в спектр от ионов $(\mathrm{OH})$. Возникновение этой полосы свидетельствует о высокой активности поверхности микрочастиц ферритов, связанной с наличием оборванных связей и, как следствие, к большой вероятности адсорбции ионами $(\mathrm{OH})^{-}$и $\mathrm{H}^{+}$ активных ОН-групп. Как было показано в работе [16], наличие ОН-групп позволяет наночастицам магнетита легко связываться с полимерными соединениями, образуя прочные защитные покрытия.

Полоса поглощения при $1630 \mathrm{~cm}^{-1}$ была интерпретирована как деформационные колебания $\delta(\mathrm{HOH})$, а полосы поглощения при $823 \mathrm{~cm}^{-1}$ и $1045 \mathrm{~cm}^{-1}$ (рис. 2), как деформационные колебания связей $\mathrm{Zn}-\mathrm{O}-\mathrm{H}$ и $\mathrm{Fe}-\mathrm{O}-\mathrm{H}$

Для всех составов ферритов в спектральном диапазоне от 50 до $1000 \mathrm{~cm}^{-1}$ наблюдаются широкие полосы поглощения, ассоциируемые с решеточными колебаниями $\mathrm{Fe}-\mathrm{O}-, \mathrm{Ni}-\mathrm{O}-$ и $\mathrm{Zn}-\mathrm{O}-$ связей. Поэтому для интерпретации полученных результатов представляет интерес информация об инфракрасных спектрах компонентов $\mathrm{ZnO}, \mathrm{NiO}$ и $\mathrm{Fe}_{2} \mathrm{O}_{3}$ (рис. 3), которые были использованы 


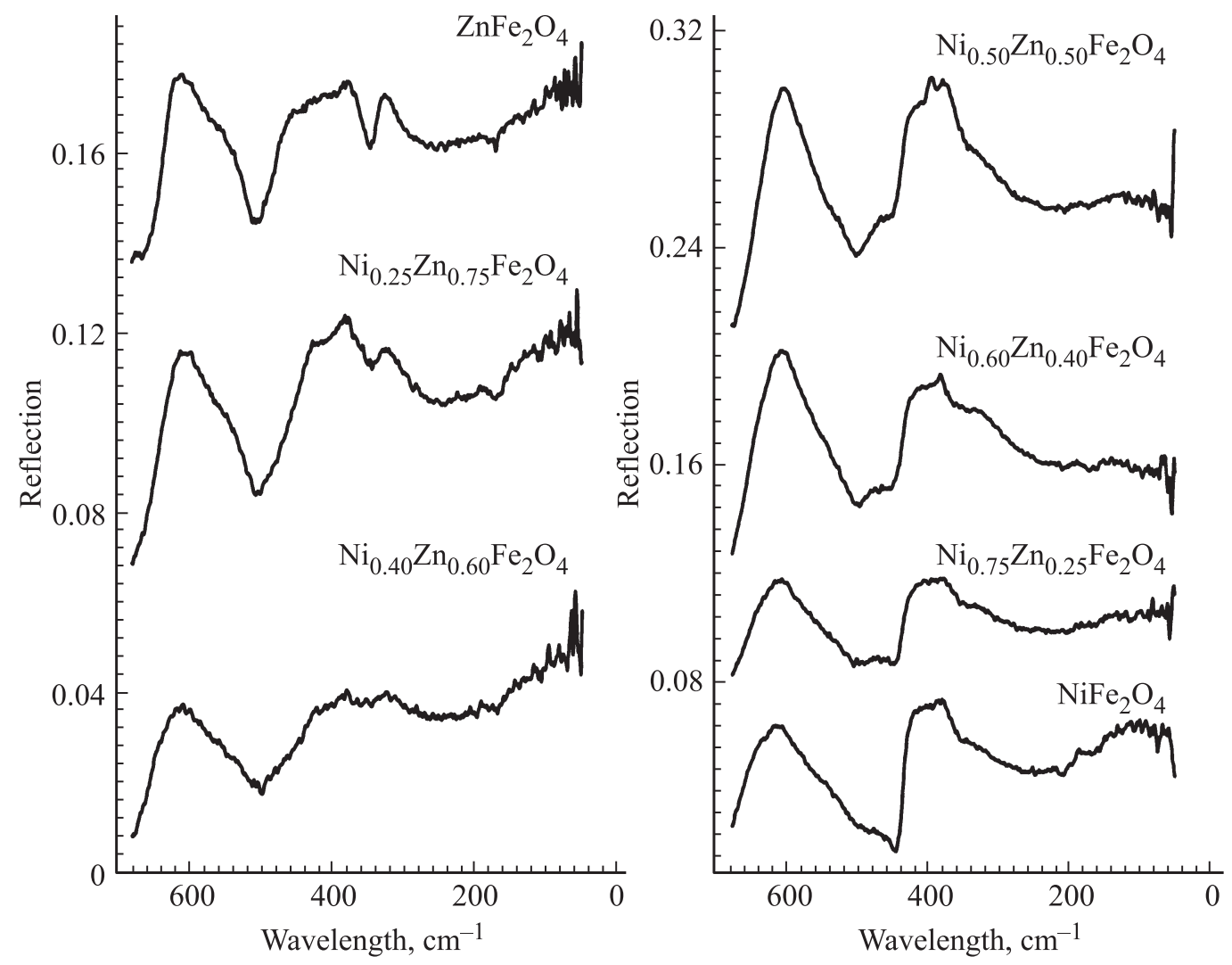

Рис. 1. Спектры диффузного тражения ферритов $\mathrm{Ni}_{1-x} \mathrm{Zn}_{x} \mathrm{Fe}_{2} \mathrm{O}_{4}$ в области $700-50 \mathrm{~cm}^{-1}$ ИК-диапазона.
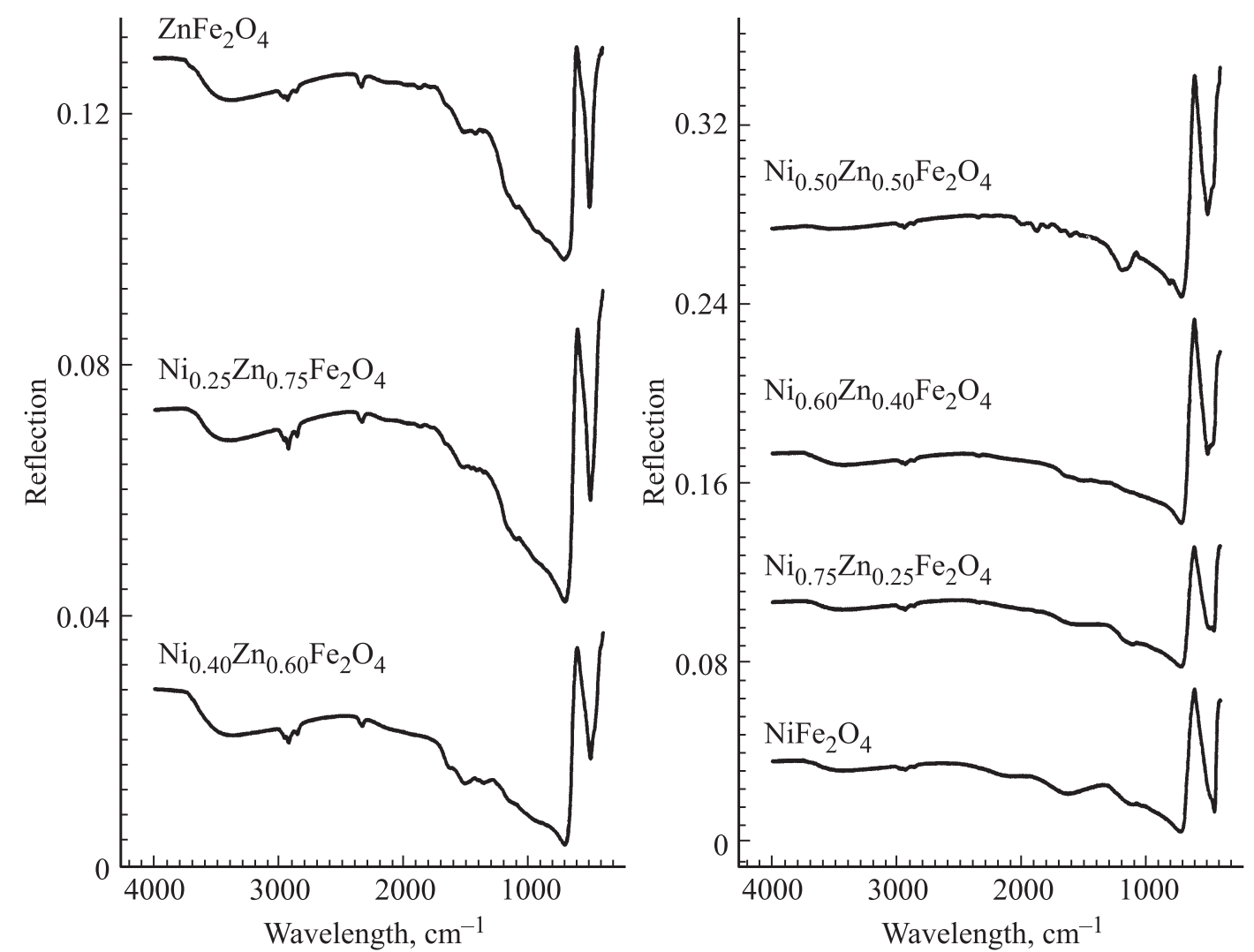

Pис. 2. Спектры диффузного тражения ферритов $\mathrm{Ni}_{1-x} \mathrm{Zn}_{x} \mathrm{Fe}_{2} \mathrm{O}_{4}$ в области $4000-700 \mathrm{~cm}^{-1}$ ИК-диапазона. 
в процессе синтеза $\mathrm{Ni}_{1-x} \mathrm{Zn}_{x} \mathrm{Fe}_{2} \mathrm{O}_{4}$-ферритов. Для сравнения на рис. 3 показан ИК-спектр $\mathrm{Ni}_{0.5} \mathrm{Zn}_{0.5} \mathrm{Fe}_{2} \mathrm{O}_{4}$.

$\mathrm{B}$ спектре $\mathrm{Fe}_{2} \mathrm{O}_{3}$ связи $\mathrm{Fe}-\mathrm{O}$ представлены характерным дублетом полос $545 \mathrm{~cm}^{-1}$ и $470 \mathrm{~cm}^{-1}$, соответственно $F_{1 u^{-}}^{1}$ и $F_{1 u^{2}}^{2}$-типов симметрии. В $\mathrm{Fe}_{3} \mathrm{O}_{4}$ аналогичные дублеты наблюдаются в положениях 590 (или 595$) \mathrm{cm}^{-1}$ и $415 \mathrm{~cm}^{-1}$ спектра [17]. Дублетная структура $\left(590 \mathrm{~cm}^{-1}\right.$ и $413 \mathrm{~cm}^{-1}$ ) наблюдается также в микропорошках $\mathrm{ZnO}$. В спектральной полосе $\left(530-430 \mathrm{~cm}^{-1}\right)$ располагается структура, соответствующая связи $\mathrm{Ni}-\mathrm{O}$ в микропорошках $\mathrm{NiO}$.

Таким образом, сравнение ИК-спектров $\mathrm{ZnO}, \mathrm{NiO}$ и $\mathrm{Fe}_{2} \mathrm{O}_{3}$ со спектром $\mathrm{NiFe}_{2} \mathrm{O}_{4}$ позволяет интерпретировать дублет $\left(604 \mathrm{~cm}^{-1}\right.$ и $\left.425 \mathrm{~cm}^{-1}\right)$, как колебания $\mathrm{Ni}-\mathrm{O}$ и $\mathrm{Fe}-\mathrm{O}$ связей соответственно. Как следует из таблицы, увеличение $x$ в составах $\mathrm{Ni}_{1-x} \mathrm{Zn}_{x} \mathrm{Fe}_{2} \mathrm{O}_{4}$ приводит к смещению линии $604 \mathrm{~cm}^{-1}$ в сторону $570 \mathrm{~cm}^{-1}$, причем при значениях $x$ вблизи 0.5 наблюдается возникновение новой компоненты $544 \mathrm{~cm}^{-1}$, соответствующей колебаниям $\mathrm{Zn}-\mathrm{O}$-связей, которая в $\mathrm{ZnFe}_{2} \mathrm{O}_{4}$ регистрируется в положении линии $542 \mathrm{~cm}^{-1}$ [9] или $569 \mathrm{~cm}^{-1}$ [10]. Заметим, что максимумы спектра около 430 и $542 \mathrm{~cm}^{-1}$, представляют собой комбинированные полосы колебаний валентных связей $\mathrm{Fe}-\mathrm{O}$ в октаэдрических позициях с ионами $\mathrm{Zn}^{2+}$ в ближайшем координационном окружении: $\mathrm{Fe}-\mathrm{O}-\mathrm{Zn}$. Положение линии $425 \mathrm{~cm}^{-1}$, интерпретированной как колебания $F_{1 u}^{2}$-типа симметрии, практически не зависит (см. таблицу) от изменения , $x$ “ в составе, что позволяет интерпретировать ее как колебания $\mathrm{Fe}-\mathrm{O}-$-вязей, то есть $\left(\mathrm{Fe}^{3+}-\mathrm{O}-2 \mathrm{Fe}^{3+}\right)$.

Зависимости интенсивностей ИК-спектров диффузного отражения от , $x$ “ в $\mathrm{Ni}_{1-x} \mathrm{Zn}_{x} \mathrm{Fe}_{2} \mathrm{O}_{4}$ интерпретировались в рамках модели, учитывающей изменения концентраций катионов $\mathrm{Fe}^{2+}[18]$ и $\mathrm{Fe}^{3+}[9]$ (рис. 4) в этих составах ферритов. Как следует из полученных результатов, изменение концентраций катионов $\mathrm{Fe}^{2+}$ и $\mathrm{Fe}^{3+} \mathrm{c}$ изменением , $x$ “ приводит к изменениям интенсивности спектров диффузного отражения, максимум которых располагается вблизи состава $x=0.6$. Вблизи этого состава концентрация катионов $\mathrm{Fe}^{2+}$ резко уменьшается и при $x=0.7$ становится равной концентрации катионов $\mathrm{Fe}^{3+}$. Заметим также, что, по-видимому, этот факт является причиной резкого роста магнитной проницаемости [2] в данном составе феррита. С другой стороны, изменение содержания катионов $\mathrm{Fe}^{2+}$ и $\mathrm{Fe}^{3+}$ в разных составах ферритов свидетельствует об изменении в них числа „перескоковых“ электронов, в результате изменения косвенного обмена [19]. Поскольку „перескоковые“ электроны согласно модели [19] формируют „свое“ магнитное поле, имеющее структуру, то изменение их концентраций влияет на общее распределение магнитного поля в феррите, что подтверждается ЭПР-исследованиями составов $\mathrm{Ni}_{1-x} \mathrm{Zn}_{x} \mathrm{Fe}_{2} \mathrm{O}_{4}$-ферритов [6]. В работе [20] было установлено наличие в ИК-спектре поглощения при температуре $300 \mathrm{~K}$ максимума при $1600 \mathrm{~cm}^{-1}$, ранее обнаруженного в спектрах Раманрассеяния, совпадающего с положением двухмагнонной
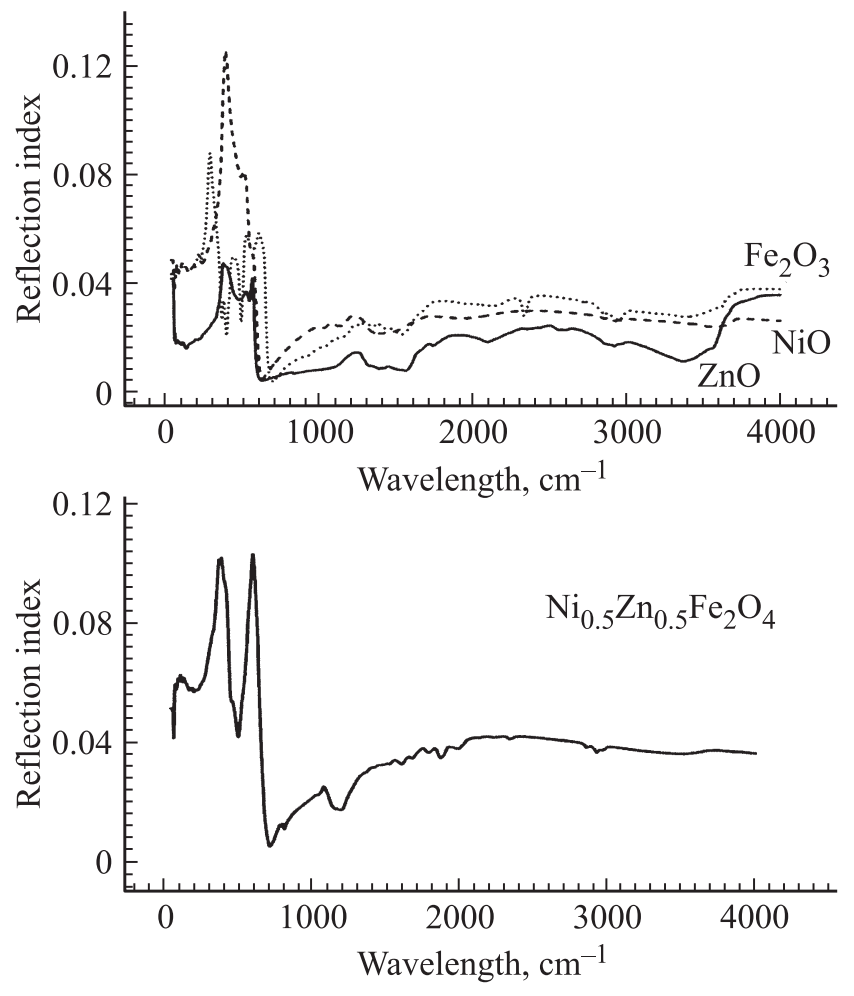

Рис. 3. ИК-спектры порошков $\mathrm{Fe}_{2} \mathrm{O}_{3}, \mathrm{NiO}$ и $\mathrm{ZnO}$, использованных в процессе синтеза ферритов $\mathrm{Ni}_{1-x} \mathrm{Zn}_{x} \mathrm{Fe}_{2} \mathrm{O}_{4}$, и выбранного для примера состава $\mathrm{Ni}_{0.5} \mathrm{Zn}_{0.5} \mathrm{Fe}_{2} \mathrm{O}_{4}$.

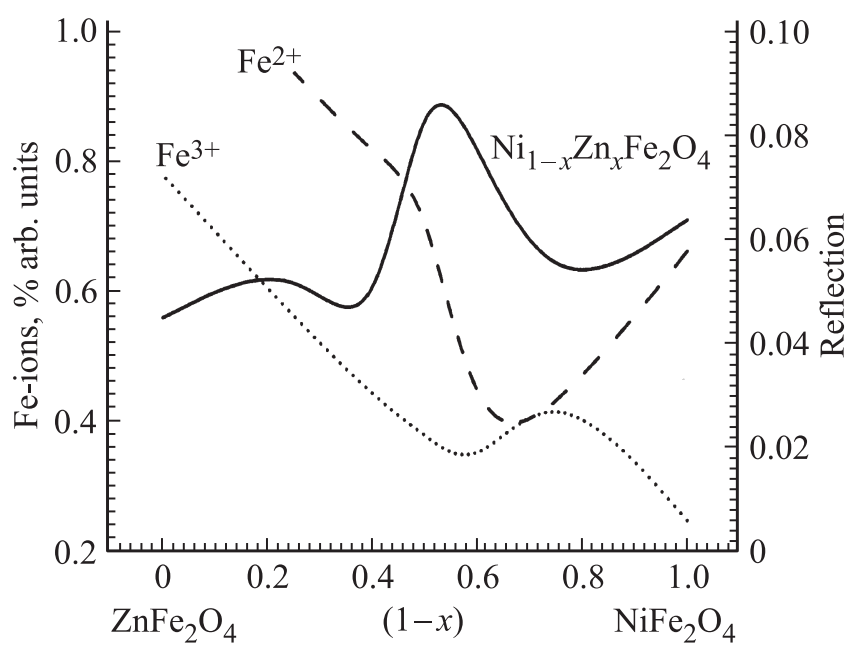

Рис. 4. Изменение интенсивности ИК-спектра отражения и концентраций катионов $\mathrm{Fe}^{2+}[16]$ и $\mathrm{Fe}^{3+}[9]$ в разных составах ферритов $\mathrm{Ni}_{1-x} \mathrm{Zn}_{x} \mathrm{Fe}_{2} \mathrm{O}_{4}$.

зоны. По предположению [20], этот максимум имеет примесный характер. В наших исследованиях в разных составах $\mathrm{Ni}_{1-x} \mathrm{Zn}_{x} \mathrm{Fe}_{2} \mathrm{O}_{4}$ положение этого максимума обнаруживается в спектральной полосе $1550-1400 \mathrm{~cm}^{-1}$, причем изменения интенсивности максимума согласуются с изменениями концентраций катионов $\mathrm{Fe}^{2+}$ и $\mathrm{Fe}^{3+}$ (рис. 4). 
Как показано в таблице, в области дальнего ИК-спектра от 300 до $50 \mathrm{~cm}^{-1}$ наблюдаются колебания типа $F_{1 u}^{3}$, возникающие между одноименными катионами. Им соответствуют спектральные линии $249 \mathrm{~cm}^{-1}\left(\mathrm{NiFe}_{2} \mathrm{O}_{4}\right)$, и $206 \mathrm{~cm}^{-1}\left(\mathrm{ZnFe}_{2} \mathrm{O}_{4}\right)$ (рис. 1). Наличие линии около $249 \mathrm{~cm}^{-1}$ во всех составах $\mathrm{Ni}_{1-x} \mathrm{Zn}_{x} \mathrm{Fe}_{2} \mathrm{O}_{4}$-ферритов свидетельствует о ее принадлежности к колебаниям $\left(\mathrm{Fe}^{3+}-\mathrm{Fe}^{3+}\right)$ связи. Соответственно, линия $206 \mathrm{~cm}^{-1}$ наблюдается только в составах, в которых присутствует $\mathrm{Zn}$. Максимум поглощения $206 \mathrm{~cm}^{-1}$ наблюдался в $\mathrm{ZnFe}_{2} \mathrm{O}_{4}$ также, например, в работе [21]. Как следует из результатов работы [18], наличие магнитного поля „перескоковых“ электронов [17] может привести к появлению антиферромагнитного резонанса в $\mathrm{Ni}_{1-x} \mathrm{Zn}_{x} \mathrm{Fe}_{2} \mathrm{O}_{4}$-ферритах, который, по оценке располагается в области $\sim 2-3 \mathrm{THz}\left(70-100 \mathrm{~cm}^{-1}\right)$. В этой области располагаются линии антиферромагнитного резонанса $\mathrm{NiO}\left(36 \mathrm{~cm}^{-1}\right)$ [12] и $\mathrm{Fe}_{2} \mathrm{O}_{3}\left(10 \mathrm{~cm}^{-1}\right)$ [22].

Заметим, что обнаруженная зависимость интенсивности ИК-спектра от, „x“ в $\mathrm{Ni}_{1-x} \mathrm{Zn}_{x} \mathrm{Fe}_{2} \mathrm{O}_{4}$, в неявной форме подразумевает наличие (перескоковый электрон) фононного взаимодействия [23], а также влияния примесных атомов на формирование ИК спектра всех составов ферритов (как было указано выше, наличие вакансий является характерной особенностью шпинелей). В частности, теоретические исследования [13] заряженной относительно кристаллической решетки $\mathrm{ZnO}$ примеси $\mathrm{Ni}^{3+}$ показали, что взаимодействие примеси с ионами ближайшего окружения должно приводить к появлению слабых по интенсивностям максимумов $3.2 \mathrm{THz}\left(460 \mathrm{~cm}^{-1}\right) ; 7.5 \mathrm{THz}$ $\left(250 \mathrm{~cm}^{-1}\right) ; 8.3 \mathrm{THz}\left(273 \mathrm{~cm}^{-1}\right) ; 9.9 \mathrm{THz} \quad\left(330 \mathrm{~cm}^{-1}\right)$; $11.2 \mathrm{THz} \quad\left(373 \mathrm{~cm}^{-1}\right) ; 12.3 \mathrm{THz} \quad\left(410 \mathrm{~cm}^{-1}\right) ; 13.8 \mathrm{THz}$ $\left(460 \mathrm{~cm}^{-1}\right) ; 15 \mathrm{THz}\left(500 \mathrm{~cm}^{-1}\right)$, среди которых обнаружены резонансное и щелевое колебания на частотах $8.3 \mathrm{THz}\left(273 \mathrm{~cm}^{-1}\right)$ и $11.2 \mathrm{THz}\left(373 \mathrm{~cm}^{-1}\right)$. Соответствующие этим колебаниям частоты приведены в таблице. Интенсивность этих колебаний, как и следовало ожидать [13], очень мала. Однако многократными исследованиями была установлена слабая зависимость ИК-спектров диффузного отражения для разных микропорошков одного состава от наличия дефектов.

\section{4. Заключение}

Исследованы ИК-спектры диффузного отражения микропорошков $\mathrm{Ni}_{1-x} \mathrm{Zn}_{x} \mathrm{Fe}_{2} \mathrm{O}_{4}$ ( $x=0,0.25,0.4,0.5$, 0.6, $0.75,1.0)$ ферритов в области от 4000 до $50 \mathrm{~cm}^{-1}$ и установлены частоты фононов в каждом составе. Показано, что наблюдаемые различия в спектрах разных составов связаны с изменением соотношения концентраций $\mathrm{Fe}^{2+}$ и $\mathrm{Fe}^{3+}$ (а также $\left.\mathrm{Ni}^{2+}, \mathrm{Ni}^{3+}, \mathrm{Zn}^{2+}\right)$ ионов. Последнее позволяет предположить, что причиной наблюдаемых различий является „слабая““ магнитная подрешетка, формируемая „перескоковыми“ электронами.

\section{Список литературы}

[1] E.W. Gorter. Philips Res. Rep. 9, 4, 295, 5, 321, 6, 403 (1954).

[2] Н.Н. Шольц, К.А. Пискарев. Ферриты для радиочастот. Энергия, М. (1966). 258 с.

[3] Ю.Г. Чекалкин, А.Е. Теплых. ФТТ 40, 1503 (1998).

[4] Ю.Н. Михайлов, В.А. Казанцев. ФТТ 52, 894, (2010).

[5] В.Г. Костишин, Б.К. Остафийчук, В.В. Мокляк, А.В. Нуриев. Материалы электрон. техники 4, 22, (2013).

[6] Sh.N. Aliyeva, Y.N. Aliyeva, A.I. Nadjafov, I.S. Hasanov, E.K. Huseynov, T.R. Mehdiyev. Phys. Status Solidi C 12, 615 (2015).

[7] В.Н. Глазков. Магнетизм. Свойства магнитноупорядоченных кристаллов, МФТИ, М. (2016). 41 с. https://mipt.ru/upload/medialibrary/f4e/13-magnets-2016_apr25.pdf

[8] Ш.Н. Алиева, А. Наджафов, Т.Р. Мехтиев. АJP Fizika XIX, 107 (2013)

[9] F. Sh. Tehrani, V. Daadmehr, A.T. Rezakhani, R.H. Akbarnejad, S. Gholipour. J. Supercond. Novel Magnetism 25, 2443 (2012).

[10] J. Wan, X. Jiang, H. Li, K.Ch. Facile. J. Mater. Chem. 22, 13500 (2012).

[11] M.K. Raju. Chem. Sci. Transact. 4, 137 (2015).

[12] Sh.G. Chou, P.E. Stutzman, Sh. Wang, E.J. Garboczi, W.F. Egelhoff, D.F. Plusquellic. J. Phys. Chem. C. 116, 16161 (2012).

[14] V.C. Farmer. The Infrared Spectra of Minerals. Mineral.Soc., London, (1974). 539 p.

[15] J.T. Keiser, C.W. Brown, R.H. Heidersbach. J. Electrochem. Soc. 129, 2686 (1982).

[16] M. Ma, Yu. Zhang, Yu. Wei, Hao-ying Shen, Haiqian Zhang, N. Gu. Colloids and Surfaces: Physicochemical and Enginering Aspects 212, 219 (2003).

[17] Z.R. Marand, M.R. Farimani, N. Shahtahmasebi. Nanomedicine J. 1, 238 (2014).

[18] S.J. Santosh, E.Sh. Sagar, B.G. Toksha, S.J. Shukla, K.M. Jadhav. Chin. J. Chem. Phys, 21, 381 (2008).

[19] К.П. Белов. УФН 166, 669 (1996).

[20] J.L.M. Vidales, A.L. Delgado, E. Vila, F.A. Lopez. J. Alloys Comp. 287, 276 (1999).

[21] J. Nishitani, K. Kozuki, T. Nagashima, M. Hangyo. Appl. Phys. Lett. 96, 221906-1 (2010).

[22] A. Kozlowski, Z. Kakol, Z. Tarnawski. Acta Phys. Pol. A 111, 537, (2007).

[23] А.Н. Кислов, В.Г. Мазуренко, А.Н. Вараксин. ФТТ 41, 618 (1999). 